\title{
State Dependent Parameter metamodelling and sensitivity analysis
}

\author{
Marco Ratto $^{\mathrm{a}, *}$, Andrea Pagano ${ }^{\mathrm{a}}$, Peter Young ${ }^{\mathrm{b}, \mathrm{c}}$ \\ a Joint Research Centre, European Commission, TP361 IPSC, via Fermi 1, 21020 Ispra (VA), Italy \\ $\mathrm{b}$ Centre for Research on Environmental Systems and Statistics, Lancaster University, Lancaster, UK \\ c Centre for Resource and Environmental Studies, Australian National University, Canberra, Australia \\ Received 13 February 2007; received in revised form 5 July 2007; accepted 16 July 2007
}

Available online 8 August 2007

\begin{abstract}
In this paper we propose a general framework to deal with model approximation and analysis. We present a unified procedure which exploits sampling, screening and model approximation techniques in order to optimally fulfill basic requirements in terms of general applicability and flexibility, efficiency of estimation and simplicity of implementation. The sampling procedure applies Sobol' quasi-Monte Carlo sequences, which display optimal characteristics when linked to a screening procedure, such as the elementary effect test. The latter method is used to reduce the dimensionality of the problem and allows for a preliminary sorting of the factors in terms of their relative importance. Then we apply State Dependent Parameter (SDP) modelling (a model estimation approach, based on recursive filtering and smoothing estimation) to build an approximation of the computational model under analysis and to estimate the variance based sensitivity indices. The method is conceptually simple and very efficient, leading to a significant reduction in the cost of the analysis. All measures of interest are computed using a single set of quasi-Monte Carlo runs. The approach is flexible because, in principle, it can be applied with any available type of Monte Carlo sample.
\end{abstract} (C) 2007 Elsevier B.V. All rights reserved.

PACS: 02.60.-x; 02.70.-c; 07.05.Tp

Keywords: Sensitivity analysis; State Dependent Parameter models; Metamodelling; High dimensional model representation

\section{Introduction}

We consider a mathematical or computational model of the form,

$Y=f\left(X_{1}, X_{2}, \ldots, X_{k}\right)=f(\mathbf{X})$,

where the input factors $X_{i}$ have a domain of variability $U$, linked to the uncertainty about their precise value. We interpret the term "factor" in a very broad sense: namely, a factor is anything that can be subject to some degree of uncertainty in the model. In global sensitivity analysis, ${ }^{1}$ all the $X_{i}$ 's are treated as random variables characterized by specified distributions, implying that the output $Y$ is also a random variable with a probability distribution, whose characterization is the object of the uncertainty analysis.

\footnotetext{
* Corresponding author.

E-mail address: marco.ratto@jrc.it (M. Ratto).

1 Sometimes also known as probabilistic sensitivity analysis.
}

The scope of sensitivity analysis (SA) is to rank the importance of the various sources of uncertainty in producing the uncertainty in the output $Y$. Variance-based sensitivity indices are the most popular measures of importance. The two key measures are the main effect

$S_{i}=V_{i} / Y(Y)=V\left[E\left(Y \mid X_{i}\right)\right] / V(Y)$

and the total effect

$S_{T_{i}}=V_{T_{i}} / V(Y)=E\left[V\left(Y \mid \mathbf{X}_{-i}\right)\right] / V(Y)$,

where $\mathbf{X}_{-i}$ indicates the array of all input factors except $X_{i}$. It can be shown [1] that these measures of importance are strictly linked to rigorously specified sensitivity settings:

FP Factor privatization: here, we look for the factor that, once "discovered" with its true value and constrained at this value, would reduce the uncertainty in the output $Y$ the most and, therefore, make the model inference more robust. The main effects provide the answer to the FP setting. 
FF Factor fixing: here, we want to identify those factors that have an irrelevant contribution to the uncertainty on the output and, therefore, can be constrained to an arbitrary value within their range of uncertainty. The total effects may be used in the FF setting.

As discussed in [2], in addition to variance/uncertainty reduction, sensitivity measures can also be interpreted in the context of regression and prediction analysis. If we were to identify the univariate function $g\left(X_{i}\right)$ of the single parameter $X_{i}$ that best approximates the original function $f\left(X_{1}, \ldots, X_{k}\right)$, assuming a quadratic loss $L=E\left(\left(f(\cdot)-g\left(X_{i}\right)\right)^{2}\right)$, then it is well known, from any standard text on statistics, that the function minimizing this loss is exactly the conditional expectation $E\left(Y \mid X_{i}\right)$. So, the best univariate approximation to the function $f(\cdot)$ is also the one whose variance provides the main effect sensitivity measure.

Jointly with the two interpretations mentioned above, two main methodological approaches are followed in the literature on the estimation of sensitivity indices: the classical approach and the metamodelling approach. Both approaches are based on Monte Carlo or quasi-Monte Carlo sampling techniques, where the uncertainty ranges of the input factors in the vector $\mathbf{X}=$ $\left[X_{1}, \ldots, X_{k}\right]$ are propagated through the computational model $Y=f(\mathbf{X})$.

The classical approach (FAST, extended FAST, Sobol', importance measures) is to estimate directly the conditional variances characterizing the sensitivity indices. Monte Carlo samples, normally generated with ad hoc designs (FAST samples, Sobol' samples, replicated Latin Hypercube samples), allow for the direct computation, in a more or less efficient manner, of the double-loop multidimensional integrals underlying the sensitivity indices. Whilst quantitatively reliable and robust in tackling the specified SA settings, these 'classical' methods have a significant computational cost, requiring thousands of model evaluations, which renders impractical their application to expensive computational models. ${ }^{2}$ According to the latest efforts at reducing the computational cost, e.g., [3], the number of model realizations required to compute the whole set of main and total effects is approximately $N \cdot k$ with $N \approx 500 \rightarrow 1000$.

In the alternative metamodelling approach, a metamodel or 'emulator' of the original computational model is estimated first; then all the measures of interest, and the mapping of $Y$, are based on this metamodel. While apparently a much more ambitious and difficult task, this approach turns out to be much more efficient than the classical alternatives. Intuitively, the higher efficiency of the metamodelling approach can be explained by the fact that it makes a better use of the information contained in the Monte Carlo sample; in particular, that information related to the smoothness of the mapping $Y$ versus the $X_{i}$ 's. This facilitates the characterization of the $Y$ behavior at any location $\mathbf{X}=\mathbf{x}^{*}$, using information derived from nearby points. In the classical methods, on the other hand, each MC run is treated independently of the rest. So the 'pattern recognition'

\footnotetext{
2 By 'expensive' we mean here models requiring several minutes, hours or even days of CPU time to perform a single run.
}

procedure of the input-output mapping, that is always implicit in metamodelling, greatly reduces the number of model evaluations needed to build an approximation to the model and, subsequently, compute sensitivities.

The general framework of metamodelling and emulation is originally formalized in [4]. Its application in SA has been demonstrated in [2], where a Bayesian approach is applied to produce a Gaussian process emulator of the computational model and, subsequently, to compute sensitivity indices in an extremely efficient way, with a computational cost of only a few hundreds of runs for a reasonable number of input variables. This computational efficiency is, however, linked to certain limitations and hypotheses: namely, that the function $f(\cdot)$ needs to be smooth and the number of input variables should not be very high $(<30)$.

In a parallel branch of research, the papers by H. Rabitz and co-workers on High Dimensional Model Representation (HDMR, Refs. [5,6]) can be seen as precursory applications of the metamodelling approach in SA. There, the metamodel is given by the so-called cut-HDMR expansion, obtained by evaluating the function $f(\mathbf{X})$ on quadrature points falling on lines, planes and hyper-planes passing through a 'base' point $\mathbf{X}_{0}$ in the input factor space, and truncating the order of the hyperplanes at a value $l<k$. Then, all the required integrals are computed on the truncated cut-HDMR function. This helps to reduce the computational cost of the analysis, albeit still not sufficiently to treat expensive models. Moreover, the cut-HDMR approach still depends significantly on the dimensionality of the problem $(k)$. Subsequently, this same research group presented the so-called Random Sampling-HDMR (RS-HDMR, see [7, 8]), where the HDMR terms are estimated by interpolating a Monte Carlo sample of the mapping $Y=f\left(X_{1}, \ldots, X_{k}\right)$ with orthogonal polynomials. These earlier references are, therefore, the first implementations of the metamodelling concept within the sensitivity context.

In this paper, we propose a new method for global SA which falls within the context of metamodelling and emulation. It is based on an approach to State-Dependent Parameter (SDP) modelling first suggested in [9], and the estimation is performed with the help of the 'classical' recursive (nonnumerical) Kalman filter [10] and associated fixed interval smoothing algorithms. In this case, the metamodel is based on a truncated ANOVA-HDMR expansion (see Section 2) and, as in the previously published approaches, it normally estimates all the main effects with only a few hundred Monte Carlo realizations $(\leqslant 1000)$, almost independently of the dimensionality $k$ of the problem. It can be used to deal with non-smooth or even discontinuous patterns in $f(\cdot)$ and an arbitrarily large number of the input factors. It can also be extended to all the interaction effects up to the second or third order.

The convergence and efficiency characteristics of metamodelling can depend strongly upon the properties of the Monte Carlo sample, which should explore the $U$ space in the best possible way. In this context, we will show that 'pre-screening' procedures, able to provide a proxy for the total effects with only an handful of model realizations (the elementary effect test, see Ref. [11]), are of crucial importance in order both to prepare 
the metamodelling analysis and to make the best use of the sequential space-filling properties of the Sobol' quasi-Monte Carlo sequences [12]. Finally, the pre-screening procedure also completes the analysis, by adding the relevant information for the FF setting, which would otherwise be missing. In fact, no metamodelling approach is yet capable of providing reliable measures of total sensitivity.

\section{HDMR and variance-based methods}

Variance-based methods are described in detail in Refs. [1, 13] and can be defined by referring to a decomposition of the function $f$ itself into terms of increasing dimensionality (High Dimensional Model Representation, HDMR, see Refs. [5,6, 14]), i.e.

$$
\begin{aligned}
& f\left(X_{1}, X_{2}, \ldots, X_{k}\right) \\
& \quad=f_{0}+\sum_{i} f_{i}+\sum_{i} \sum_{j>i} f_{i j}+\cdots+f_{12 \ldots k},
\end{aligned}
$$

where each term is a function only of the factors in its index, i.e. $f_{i}=f\left(X_{i}\right), f_{i j}=f\left(X_{i}, X_{j}\right)$ and so on. The various terms are defined as follows:

$f_{0}=E(Y)$,

$f_{i}=E\left(Y \mid X_{i}\right)-f_{0}$,

$f_{i j}=E\left(Y \mid X_{i}, X_{j}\right)-E\left(Y \mid X_{i}\right)-E\left(Y \mid X_{j}\right)-f_{0}$,

i.e. as discussed in Section 1, they provide the best approximation to $f(\cdot)$ in a least squares sense. If the input factors are independent, all the terms of the decomposition are orthogonal and the decomposition (3) is unique.

It is obvious that $V\left(f_{i}\right) / V=V\left(E\left(Y \mid X_{i}\right)\right) / V$; in other words, the main effect (1) equals the variance of the first order term of the HDMR decomposition (ANOVA-HDMR), normalized by the unconditional variance $V$. For independent input factors, a decomposition scheme of the total unconditional variance $V(Y)$, equivalent to HDMR, can be derived $[14,15]$

$V(Y)=\sum_{i} V_{i}+\sum_{i} \sum_{j>i} V_{i j}+\cdots+V_{12 \ldots k}$,

where

$V_{i}=V\left[E\left(Y \mid X_{i}\right)\right]$,

$V_{i j}=V\left[E\left(Y \mid X_{i}, X_{j}\right)\right]-V_{i}-V_{j}$,

$V_{i j l}=V\left[E\left(Y \mid X_{i}, X_{j}, X_{l}\right)\right]-V_{i j}-V_{i l}-V_{j l}-V_{i}-V_{j}-V_{l}$.

Normalizing by $V$ from Eq. (5), we obtain the closed identity for sensitivity indices:

$1=\sum_{i} S_{i}+\sum_{i} \sum_{j>i} S_{i j}+\cdots+S_{12 \ldots k}$.

The sensitivity indices are nicely scaled in the range $[0,1]$ and, for the main effects, we have $\sum_{i} S_{i} \leqslant 1$, where the equality holds for purely additive models.
For independent inputs, the total effect (2) can also be defined as the sum of all effects containing the factor $X_{i}$, i.e.

$S_{T_{i}}=S_{i}+\sum_{j>i} S_{i j}+\sum_{l>j>i} S_{i j l}+\cdots+S_{12 \ldots k}$.

This explains the difficulty of building a metamodel that provides an accurate estimation of the total indices. Unless only a few, low order interaction terms are present, it is very easy to miss some of these terms.

\section{The method}

We estimate the terms in the ANOVA-HDMR decomposition using a special recursive fixed interval smoothing algorithm that estimates the parameters in a State-Dependent Parameter (SDP) formulation of the input-output mapping. The methodology presented in this paper resembles ideas and tools from signal processing and time series analysis: in particular, it exploits an approach to non-stationary and non-linear signal processing based on the identification and estimation of stochastic models with time variable (TVP) or state dependent (SDP) parameters (see Refs. [16-18] for a description of the method and a full list of references on the background to its development).

\subsection{Sampling strategy}

A particularly important aspect of any metamodelling exercise concerns the sampling strategy. In our opinion, two main requirements have to be fulfilled: (i) the sample must have optimal space-filling properties, (ii) the space-filling properties must be maintained when the metamodelling process is performed in a sequential manner. The first requirement is connected to the efficiency of the estimation process, helping to ensure that a representative metamodel requires the minimum number of model runs. The second requirement ensures that the optimal space filling properties are maintained in sequential processing, where the analyst adds new realizations within a metamodelling exercise, in order to improve the model.

In most quasi-Monte Carlo computations, low-discrepancy sequences are used because they provide the best convergence properties: for example, the $L P_{\tau}$ sequences of Sobol' [12,19] or Niederreiter [20]. Such sequences can be computed in a "superfast" way [21] and can be generated by one subroutine. Indeed, some low-discrepancy samples can provide optimal characteristics: for example, the Latin Supercube Generator (LSS, Ref. [22]) or Latin Hypercube samples coupled with some optimization algorithm (maximin LHs), as in [2]. Such optimal properties are achieved at a given sample size, but sequentiality is lost. Moreover, they require the implementation of computationally intensive algorithms. ${ }^{3}$

The topic of convergence properties and comparisons between quasi-Monte Carlo and Monte Carlo methods has been

\footnotetext{
3 In order to reduce the computational load, in Ref. [2] a suboptimal procedure is applied, based on iterative acceptance-rejection of permutations of point co-ordinates from a base LHs sample.
} 
extensively investigated. A conclusion from Ref. [23] is that "No one of the sequences [...] can always be regarded as superior to the others. Qualification of this kind depends strongly on the problem considered". The main advantage of a quasiMonte Carlo approach is its potential for improving the convergence characteristics. The rate of stochastic convergence for pure random Monte Carlo methods is $N^{-1 / 2}$ while, for some applications, the actual rate of convergence can be $N^{-\alpha}$ with $\alpha \leqslant 1$ [21]. Moreover, " . . . it is not correct that the application of quasi-Monte Carlo is expedient only for relatively low dimension $k \leqslant 12$ or 15 " [21]. A rather spectacular example of quasi-Monte Carlo, using the Sobol' sequence at $k=360$, is from finance mathematics, as described in Ref. [24], where it is clearly superior to Monte Carlo.

In this paper, we propose the use of Sobol' sequences because of their simplicity and because we attach a high value to sequentiality. Moreover, since quasi-Monte Carlo sequences are deterministic, we can exploit the known properties of the Sobol' samples to make the best use of them. More specifically, we take advantage of the property that, if the input factors are presorted in such a way that the most important ones correspond to the first columns of the Sobol' sequence, optimal convergence rates $\left(\approx N^{-\alpha}\right.$, with $\alpha$ approaching 1$)$ can be achieved [23,25].

\subsection{Factor screening and pre-sorting}

In order to perform the required pre-sorting, we apply the elementary effect test (EET, Ref. [11]), which is an extension and modification of [26]. The method varies one factor at a time across a certain number of levels selected in the space of the input factors. For each variation, the elementary effect for the factor is computed as the incremental ratio:

$$
\begin{aligned}
e e_{i}= & \frac{Y\left(x_{1}, x_{2}, \ldots, x_{i}+\Delta x_{i}, \ldots, x_{k}\right)}{\Delta x_{i}} \\
& -\frac{Y\left(x_{1}, x_{2}, \ldots, x_{i}, \ldots, x_{k}\right)}{\Delta x_{i}} .
\end{aligned}
$$

A number, $r$, of stepwise trajectories are used to scan the factor space, where each curve consists of $k+1$ points that allow for the computation of one elementary effect for each of the $k$ input factors. In total, for each factor, $r$ different estimates of elementary effects $e e_{i}$ are computed. Then the elementary effect tests $E E T_{i}$ are computed, as the mean of the absolute values $\left|e e_{i}\right|$ over the $r$ repetitions $E E T_{i}=1 / r \sum_{1}^{r}\left|e e_{i, r}\right|$. The $E E T_{i}$ 's are used to rank the importance of the factors. Here, $E E T_{i}$ has similarities with the $S_{T_{i}}$ index, in the sense that it tends to produce a ranking of the factors very similar, or identical, to that based on the $S_{T_{i}}$ indices, but at an extremely small computational cost (of the order $8 \cdot(k+1)$, as a rule of thumb). In a similar manner to the total index, $E E T_{i}$ does not distinguish between main effects and interactions, but highlights the importance of an input factor regardless of the effect type.

To summarize, performing a small number of additional model runs to compute the $E E T_{i}$ 's, has the following advantages: it can
1. effectively treat the FF setting, adding fundamental information to the whole analysis;

2. make the best use of the simple quasi-random sampling strategy, by exploiting optimally its sequential, space filling properties;

3. reduce the dimensionality of the metamodelling process by eliminating the factors flagged as unimportant by the $E E T_{i}$ 's.

This latter aspect would also be able to offset the dimensionality limitation of the Gaussian emulator in [2].

\subsection{Setting up the SDP Model for HDMR estimation}

Let us start by considering the first order HDMR representation of the computational model,

$$
Y_{t}-f_{0}=f_{1}\left(X_{1, t}\right)+f_{2}\left(X_{2, t}\right)+\cdots+f_{k}\left(X_{k, t}\right)+o\left(\mathbf{X X}^{\prime}\right) .
$$

This can be considered as a State Dependent Regression (SDR) model (see Ref. [17], p. 135 et seq.), i.e.:

$$
\begin{aligned}
& Y_{t}-f_{0}=\mathbf{X}_{t}^{\mathrm{T}} \mathbf{p}_{t}+e_{t} \\
&=p_{1, t} X_{1, t}+p_{2, t} X_{2, t}+\cdots+p_{k, t} X_{k, t}+e_{t}, \\
& e_{t}=N\left(0, \sigma^{2}\right),
\end{aligned}
$$

where we may assume that all terms of high order can be approximated by a Gaussian white noise process with zero mean and variance $\sigma^{2}$, i.e. the truncated HDMR is viewed as a stochastic non-linear system. This can be justified by a version of the central limit theorem [27], since the truncated terms can be considered as the sum of a large number of independent random variables with zero mean and arbitrary probability distribution. ${ }^{4}$ We adopt here the time series notation, but with the index $t$ spanning the sequence of Monte Carlo evaluations, $t=1, \ldots, N$.

Each State Dependent Parameter (SDP) $p_{i, t}$ in the SDR (10) is a function of the corresponding input factor $X_{i, t}$. If we compare these SDPs with the corresponding $f_{i}\left(X_{i, t}\right)$ in (9), then $f_{i}\left(X_{i, t}\right)=p_{i, t} X_{i, t}$. Estimating the SDPs $p_{i, t}$ is, therefore, equivalent to providing a mapping of the first order terms of the HDMR decomposition (9). While intuitively clear, the formulation (10) can have singularity problems when the support of $X_{i}$ contains a zero. This could be avoided by introducing a rank transformation of $X_{i}$, shifted by a constant (e.g., 1). However, the SDR parameters may then lose their 'physical' meaning, so it is probably better to simply reformulate the problem by introducing constant unity regressors: i.e.

$Y_{t}-f_{0}=\mathbf{1}_{t}^{\mathrm{T}} \mathbf{p}_{t}+e_{t}=p_{1, t}+p_{2, t}+\cdots+p_{k, t}+e_{t}$,

$e_{t}=N\left(0, \sigma^{2}\right)$,

where now $f_{i}\left(X_{i, t}\right)=p_{i, t}$.

In order to estimate the $p_{i, t}$, it is necessary to characterize the variability of $p_{i, t}$ in some stochastic manner. In general this

\footnotetext{
4 These terms are as many as $2^{k}-1-k$.
} 
is accomplished by assuming that the evolution of each SDP can be characterized by one member of the Generalized Random Walk (GRW) class on non-stationary random sequences (see, e.g., Refs. [28,29]). In the present context, for instance, the integrated random walk (IRW) process turns out to provide the best results because it ensures that the estimated SDP relationship has the smooth properties of a cubic spline. Given this IRW characterization of the $p_{j, t}$ 's, either of the SDR models (10) or (11) can then define the observation equation in an overall State-Space (SS) formulation of the form:

$$
\begin{array}{ll}
\text { Observation equation: } & Y_{t}=\mathbf{Z}_{t} \mathbf{p}_{t}+e_{t}, \\
\text { State equations: } & p_{i, t}=p_{i, t-1}+d_{i, t-1}, \\
& d_{i, t}=d_{i, t-1}+\eta_{i, t},
\end{array}
$$

where $\mathbf{Z}_{t}$ is either $\mathbf{X}_{t}$ as in (10) or $\mathbf{1}_{t}$ as in (11); while $e_{t}$ and $\eta_{i, t}, i=1,2, \ldots, k$, are zero mean white noise inputs with variance $\sigma^{2}$ and $\sigma_{\eta_{i, t}}^{2}$, respectively. Here, the $\eta_{i, t}$ ('system disturbances' in systems terminology) provide the stochastic stimulus for parametric change in the model and they are assumed to be independent of each other (i.e. their covariance matrix $\mathbf{Q}$ is purely diagonal) and independent of the observation noise $e_{i, t}$.

Given this state space formulation of the SDP model, it seems straightforward, at first sight, to estimate the SDPs, which are surrogate state variables in the model (12), using the recursive Kalman Filter (KF) and an associated recursive Fixed Interval Smoothing (FIS) algorithm (see, e.g., Refs. [10,30]). The latter FIS algorithm then yields an estimate $\hat{p}_{i, t \mid N}$ of $p_{i, t}$ at each data sample, where $N$ is the number of data samples (here, Monte Carlo realizations). However, the fact that no 'temporal' order actually exists in the present context might imply serious problems for recursive state estimation based on the above model (12), which is formulated in normal time series terms, with $t$ denoting the temporal order.

Fortunately, this lack of inherent temporal ordering in the Monte Carlo realizations is not a problem because we know that the parameters are state dependent. Consequently, the data can be re-ordered so that the parameters can be well estimated under the assumption that they are varying in a manner that satisfies the IRW model assumption in the sorted space. Moreover, it is logical that this sorting of the data for each parameter $p_{i, t}$ should be carried out with respect to the corresponding input factor $X_{i, t}$, on which the parameter is assumed to depend. Since each SDP is dependent on a different input factor, however, it requires a different sorting strategy to the other SDPs. Hence the 'backfitting' procedure, as described in Refs. [16,17], is exploited.

At each iteration of this backfitting algorithm, it is necessary to optimize the hyper-parameters associated with the state space model (12), namely the white noise variances $\sigma^{2}$ and $\sigma_{\eta_{i, t}}^{2}$. In fact, by a simple reformulation of the KF and FIS algorithms, each SDP and its stochastic IRW process model can entirely characterized by one Noise Variance Ratio (NVR) hyper-parameter, where $\mathrm{NVR}_{i}=\sigma_{\eta_{i, t}}^{2} / \sigma^{2}$. These NVR values are, of course, unknown a priori and need to be optimized: for example, in the above references, this is accomplished by maximum likelihood optimization using prediction error de- composition [31]. If any NVR estimate obtained in this manner is insignificantly different from zero, then no pattern is actually recognized, implying a flat $f_{i}$ term, i.e. a negligible main effect for factor $X_{i}$.

The Gaussian hypothesis for input and observation white noise inputs is not essential, since the above procedure provides an optimal linear predictor for $Y$ because the estimates of the $p_{i, t}$ (i.e. $\left.\hat{p}_{i, t \mid N}=\hat{f}_{i}\left(X_{i, t \mid N}\right)\right)$ are obtained by orthogonal projections with respect to the observation noise $e_{i, t}$ (i.e. all other HDMR terms), perfectly matching the orthogonality condition of HDMR. Moreover, under the Gaussian hypothesis, the linear predictor becomes exactly the conditional expectation, as required in (1). At the same time, the recursive filtering and smoothing procedures also provide, in a natural way, standard errors of the estimated state dependent parameters, that allow for testing the relative significance of the FIS estimates $\hat{p}_{i, t \mid N}$ and, hence, the estimated $f_{i}\left(X_{i, t \mid N}\right)$ patterns.

In order to get the flavor of the SDP estimation results, let us consider a simple function

$Y=X_{1} \cdot X_{2}+X_{3}$

with

$X_{i} \sim U(-1,1)$

and perform an analysis using a Sobol' $L P_{\tau}$ sample of dimension 256. This model has only one non-zero main effect for $X_{3}$ and one 2 nd order interaction term for $\left(X_{1}, X_{2}\right)$. In Fig. 1 we show the SDP estimation of the first order HDMR terms $f_{1}, f_{2}$ and $f_{3}$. We also report the estimated $95 \%$ error band, indicating high significance of the estimation for $f_{3}$.

The algorithm for SDP model estimation can be seen as a special form of non-parametric estimation and, as such, can be compared with other non-parametric methods, such as the Generalized Additive Modelling (GAM) approach of Ref. [32]. However, in both conceptual and algorithmic terms, the SDP approach is significantly different from this earlier approach and seems more appropriate to the estimation of nonlinear, stochastic models. Moreover, the recursive methodology, on which SDP estimation is based, is couched in optimal maximum likelihood estimation terms that seem more elegant and flexible than the scatter-plot smoothing procedures used by Hastie, Tibshirani and others. Nonetheless, the backfitting procedure used here is similar to that used in GAM and so its convergence properties are similar to those of the GAM algorithms (see, e.g., [32]).

It should also be noted that, in the above SDR model estimation procedure, the stochastic model for each SDP can be based on any member of the GRW family and is not limited to the IRW model. For instance, Random Walk (RW) or Smoothed Random Walk (SRW) models might be identified as being preferable in certain circumstances because they yield less smooth estimates. Indeed, if any sharp changes or jumps seem possible following any initial identification phase in the analysis, then these can be handled using 'variance intervention' (see Ref. [28]), i.e. the NVR at some specific locations is allowed to assume a large value in order to follow sharp changes in $Y$. Moreover, in the case of heteroscedastic behavior (i.e. 
(a)

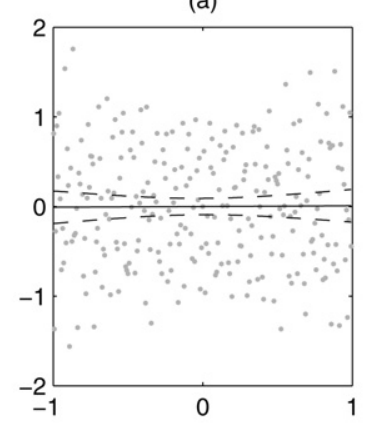

(d)

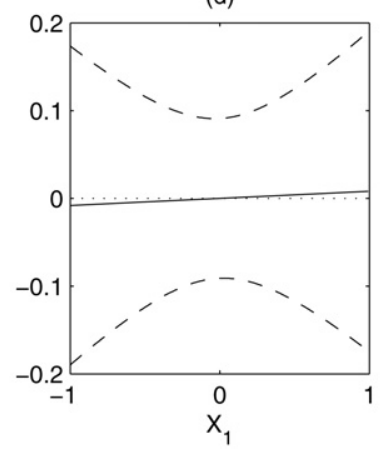

(b)

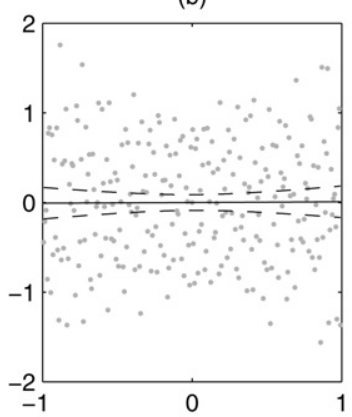

(e)

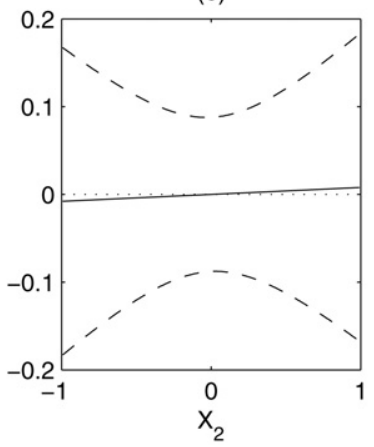

(c)

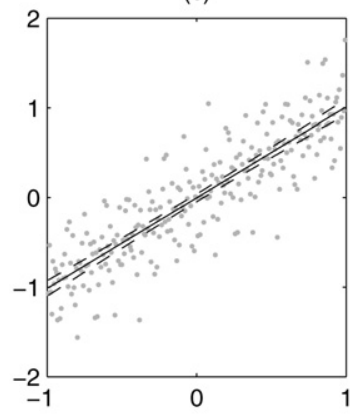

(f)

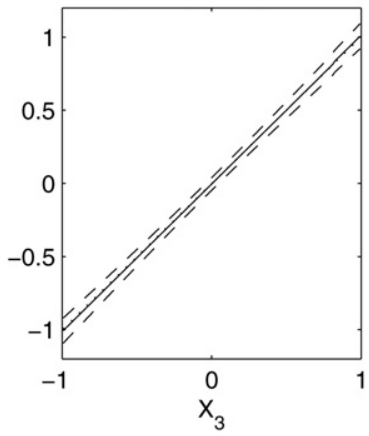

Fig. 1. SDR estimation of the first-order HDMR of the simple model (13). (a)-(c) Scatter plots of $Y$ versus $X_{i}$ (grey dots), with the smoothed estimates of the $f_{i}+f_{0}$ functions (solid lines) and their 95\% error bands (dashed lines). (d)-(f) Detail of the estimated $f_{i}$ functions (solid lines) with estimated $95 \%$ error bands (dashed lines) and analytic $f_{i}$ functions (dotted lines).

change in variance) in the observation noise, this can be further modelled in a SDP manner, e.g., by making the NVR a function of some state (see Refs. [33,34]). In the specific case discussed here, the NVR will simply depend on $X_{i}$. This can then be inserted easily into the KF and FIS algorithms. In practice, these extensions enable the SDR models to adapt to such situations, typical in non-linear systems, where one single, constant 'smoothing parameter' NVR does not allow us to follow appropriately the observed patterns of the $f(\cdot)$ mapping.

\subsubsection{Extension to interaction terms}

Considering the analogies of SDR model estimation with signal extraction and its associated frequency domain considerations, the extension of the SDP approach to the estimation of the interaction terms in the HDMR requires a generalization of the ordering concept applied for the main effects. For example, in order to estimate a second order effect $V_{i j}$, we need to define a sorting strategy that implies a low frequency characteristic for the couple $\left(X_{i}, X_{j}\right)$ of input factors under consideration, while all other combinations have a 'white' spectrum. From this, as for the main effects, a smoothing procedure on the ' $2 D$-sorted' output signal $Y$ will allow us to separate a low frequency component, attributable to the couple $\left(X_{i}, X_{j}\right)$, from the remainder of the $Y$ spectrum. Moreover, since each interaction effect will have a different sorting, the backfitting procedure has to be exploited for the interaction effects as well.

In order to visualize this, let us consider again the simple model (13) and perform the analysis of the second order in-

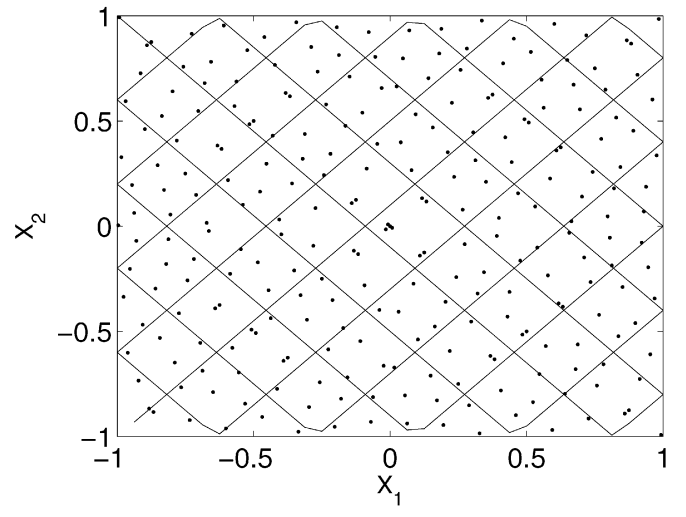

Fig. 2. Sorting trajectory in the $\left(X_{1}, X_{2}\right)$ plane.

teraction term $\left(X_{1}, X_{2}\right)$. Since we want to compute the $V_{12}$ interaction effect, the $2 D$ sorting requires exploration of the $\left(X_{1}, X_{2}\right)$ plane along a closed trajectory, like the one shown in Fig. 2, with the sorting of the sample points carried out as they fall within the band delimited by two adjacent lines. This allows for the identification of an ordering where $\left(X_{1}, X_{2}\right)$ has low frequency characteristics, while $X_{3}$ maintains the white spectrum (Fig. 3). The corresponding sorted output signal $Y$ can then be analyzed to identify the 2nd order interaction term (Fig. 4).

Considering the above procedure within the SDP formalism, the HDMR terms of order higher than one can be expressed as: 

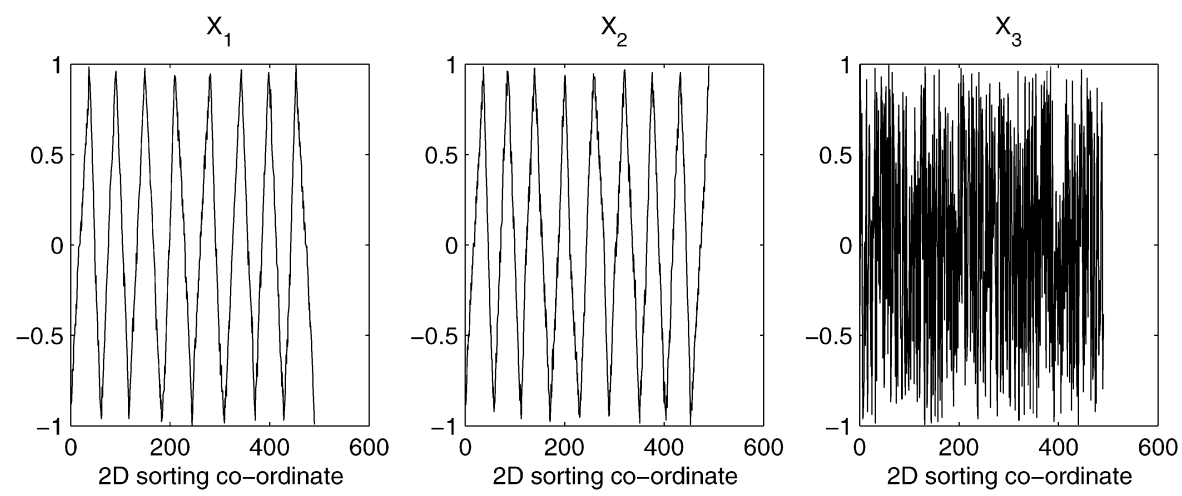

Fig. 3. Sorted sample used to compute the $\left(X_{1}, X_{2}\right)$ interaction.
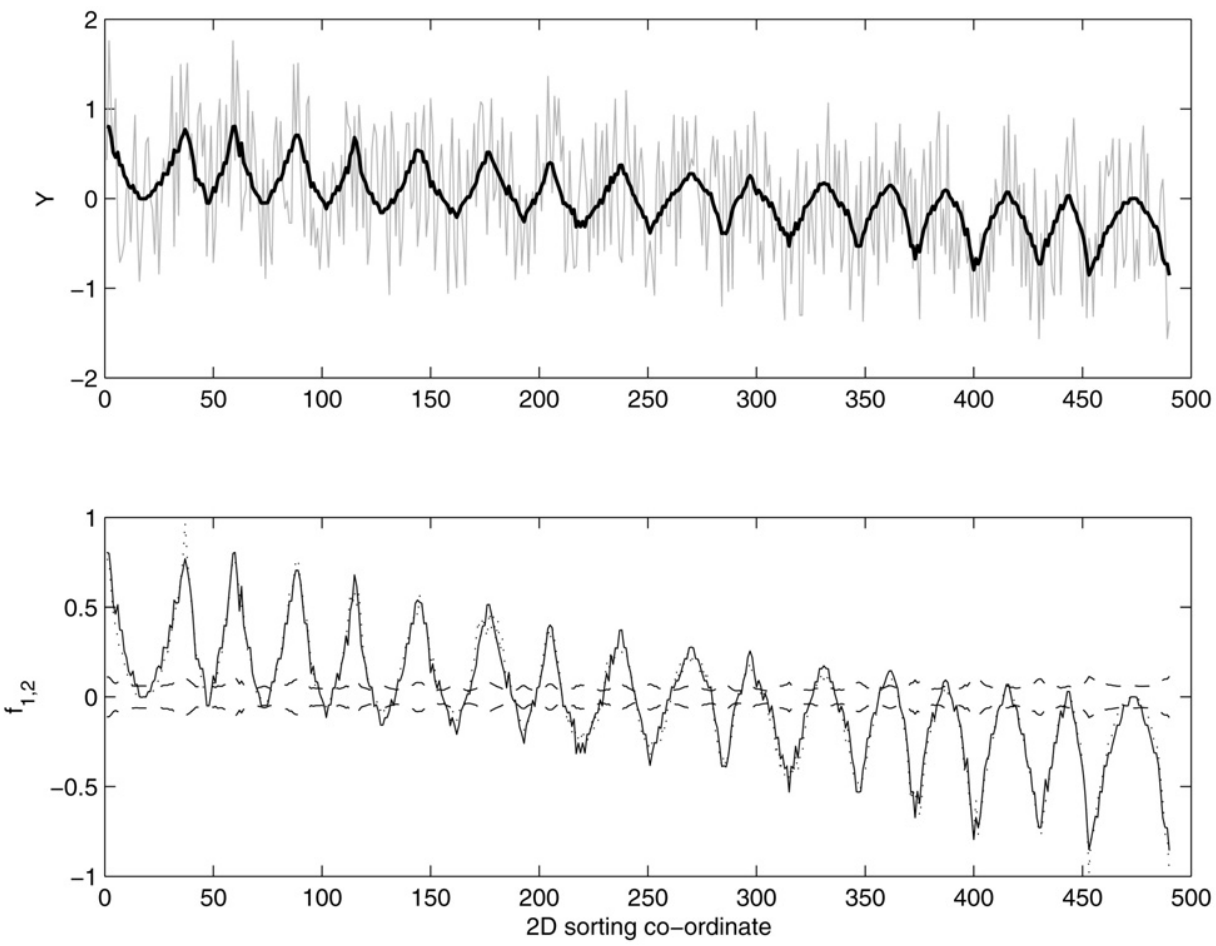

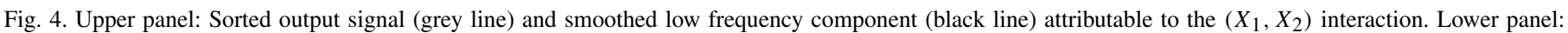

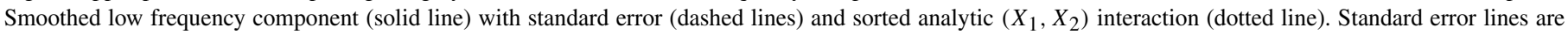
plotted around the zero line for ease of reading.

$\sum_{j>i} p_{i j, t}\left(s_{i j}\right)+\sum_{l>j>i} p_{i j l, t}\left(s_{i j l}\right)+\cdots+$ h.o.t.,

where each state-dependent parameter $p_{I, t}\left(s_{I}\right), I=i_{1}, \ldots, i_{l}$, follows a stochastic IRW process and depends on a state variable $s_{I}$ that moves according to the generalized ordering strategy along the co-ordinates of the group of factors indexed by $I$. In the test example discussed later in Section 4 of this paper, the HDMR decomposition is always truncated at the second order and the portion of variance left out by this truncation will always be specified.

The definition (14) can be further generalized by assuming that the interaction terms are made of a parametric regressor $\psi_{I}\left(\mathbf{X}_{I}\right)$, e.g., like those obtained by following the RSHDMR approach $[7,8]$, multiplied by a state-dependent parameter:

$$
\begin{aligned}
& \sum_{j>i} p_{i j, t}\left(s_{i j}\right) \cdot \psi_{i j}\left(X_{i, t}, X_{j, t}\right) \\
& \quad+\sum_{l>j>i} p_{i j l, t}\left(s_{i j l}\right) \cdot \psi_{i j l}\left(X_{i, t}, X_{j, t}, X_{l, t}\right)+\cdots+\text { h.o.t. }
\end{aligned}
$$

The generic regressor $\psi_{I}\left(\mathbf{X}_{I}\right)$ can be made of simple expressions like monomials. For example, in a second order term, we can take $\psi_{i j}\left(X_{i, t}, X_{j, t}\right)=X_{i, t} \cdot X_{j, t}$ and the interaction term takes the simple form $p_{i j, t}\left(s_{i j}\right) \cdot X_{i, t} \cdot X_{j, t}$. This generalized formulation shows how the SDP approach introduces a significant degree of flexibility by allowing the regression coefficients in a 'standard' parametric model approximation to be state dependent, in order to follow more precisely local curvature and non-linearity, which might otherwise require the use of high order basis expansions in the full parametric RS-HDMR. 
In this context, an efficient use of RS-HDMR in combination with SDP modelling is to apply a low order basis expansion for the RS-HDMR (e.g., second-order polynomials) and refine such a 'coarse' polynomial regression with SDP, where significant corrections are identified according to the ML estimation of the NVR's. Subsequently, this non-parametric correction can guide the choice of the most suitable basis for a full parametric model approximation (e.g., RBF instead of polynomials) that best accounts for the SDP estimation results.

\subsubsection{Extension to dependent inputs}

The extension of the present metamodelling methodology to the case when the input factors are not independent is straightforward. In this case, the expressions (3)-(4) can still be written, but its uniqueness is lost, while the variance decomposition (5) no longer holds. Therefore, the decomposition into additive functional components which are no longer unique can be judged of less use in the present context. However, using the same generalized sorting strategy described before, one can still estimate single conditional expectation terms:

$f_{I}^{c}=E\left(Y \mid \mathbf{X}_{I}\right)$

as

$Y_{t}=p_{I, t}\left(s_{I}\right)+e_{t}, \quad e_{t} \sim N\left(0, \sigma^{2}\right)$.

In Eq. (16), the $c$ exponent indicates that the functional approximation for the group of factors $I=i_{1}, \ldots, i_{l}$ is 'closed' over the entire set of contributions from order 1 to order $l$ within the group. In this case, the estimation of (17) shows the overall degree of explanation of the output $Y$ provided by the group of factors indexed by $I$. Indeed, the quantity $V\left(f_{I}^{c}\right) / V(Y)$ is well known in statistics as 'correlation ratio' or 'non-parametric $R^{2}$, as a measure of the explanatory power of covariates in regression.

\subsubsection{Estimating sensitivity indices}

Once the smoothed estimates of the HDMR terms $\hat{f}_{I}$ have been obtained, the estimation of sensitivity indices $S_{I}=$ $V\left[E\left(Y \mid \mathbf{X}_{I}\right)\right] / V(Y)$ is straightforward. As discussed in [35], three estimators can be applied for this purpose. Given the size $N$ of the Monte Carlo sample, the estimator used here is:

$\hat{S}_{I}=\frac{N^{-1} \sum_{s=1}^{N}\left(\hat{f}_{I}\left(\mathbf{x}_{I, s}\right)-\bar{f}\right)^{2}}{\sigma_{Y}^{2}}$,

where $\bar{f}=N^{-1} \sum \hat{f}_{I}\left(\mathbf{x}_{I, S}\right)$ and $\sigma_{Y}^{2}=N^{-1} \sum\left(y_{S}-\bar{Y}\right)^{2}$. In [35] the error estimate for (18) is also provided, by showing that $N^{1 / 2}\left(\hat{S}_{I}-S_{I}\right)$ is asymptotically normal with mean zero and variance $\left(1-S_{I}\right)^{2} V\left[y^{* 2}-u^{2}\right]$, where $y^{*}$ and $u$ are the standardized output and residual, respectively, i.e. $y_{s}^{*}=\left(y_{s}-\right.$ $\left.\mu_{Y}\right) / \sigma_{Y}$ and $u_{S}=\left(y_{S}-\hat{f}_{I}\left(\mathbf{x}_{I, S}\right)\right) /\left(\sigma_{Y}\left(1-\hat{S}_{I}\right)^{1 / 2}\right)$. Hence, the standard error of the estimate of $S_{I}$ is given by:

$\operatorname{SE}\left(\hat{S}_{I}\right)=\left(1-\hat{S}_{I}\right) \cdot \operatorname{std}\left[y_{s}^{* 2}-u_{s}^{2}\right] / N^{1 / 2}$.

\subsection{Parameterization}

The metamodelling exercise is completed by parameterizing the HDMR terms estimated in the SDR

$$
\begin{aligned}
Y & =f\left(X_{1}, \ldots, X_{k}\right) \\
& =f_{0}+\sum_{1 \leqslant i \leqslant k} f_{i}\left(X_{i}\right)+\sum_{1 \leqslant i<j \leqslant k} f_{i j}\left(X_{i j}\right) \text { h.o.t. }
\end{aligned}
$$

The HDMR representation is associated with the decomposition of the space of square integrable functions $L^{2}(\operatorname{supp}(f), \mu)$, where $\mu$ is a probability measure on $\operatorname{supp}(f)$. Under the assumption of independent input factors, this is equivalent to decomposing $\operatorname{supp}(f)$ into a product space and $L^{2}$ as a direct sum.

Since each HDMR term is square-integrable, we can write $f$ as

$$
\begin{aligned}
f\left(X_{1}, \ldots, X_{k}\right)= & f_{0}+\sum_{1 \leqslant i \leqslant k} \sum_{n=1}^{\infty} \alpha_{n}^{i} u_{n}^{i} \\
& +\sum_{1 \leqslant i<j \leqslant k} \sum_{n_{1}+n_{2}=2}^{\infty} \alpha_{n_{1} n_{2}}^{i j} u_{n_{1}}^{i} \otimes u_{n_{2}}^{j}+\cdots,
\end{aligned}
$$

where $\left\{u_{n}^{i}\right\}_{n \in \mathbb{N}}$ is an orthonormal basis of $L^{2}\left(\operatorname{supp}\left(X_{i}\right), \mu_{i}\right)$. As far as the variance is concerned, it is straightforward to show that if $f_{i}=\sum_{n=1}^{\infty} \alpha_{n}^{i} u_{n}^{i}$, then $\operatorname{var}\left(f_{i}\right)=\sum_{n=1}^{\infty}\left(\alpha_{n}^{i}\right)^{2}$. Let us suppose that $f_{i}$ does not significantly contribute to model's output, i.e. $\left|f_{i}\right|^{2}<\varepsilon$. If we compute the variance, we obtain $\operatorname{var}\left(f_{i}\right)<\varepsilon$, which implies that all the $\alpha_{n}^{i}$ coefficients are negligible and, therefore, there is no need to parameterize $f_{i}$.

Using the SDR estimation results, for each sample size $N$, we denote by $\hat{f}_{i}(N)$ the non-parametric approximation of $f_{i}$. Note that, once the sample size $N$ has been selected, the functional elements become $\mathbb{R}^{N}$ vectors. For each $\hat{f}_{i}(N)$ define

$\hat{f}_{i}^{M}(N)=\sum_{n=1}^{M} \alpha_{n}^{i} u_{n}^{i}(N)$

so that

$\lim _{M \rightarrow \infty} \hat{f}_{i}^{M}(N)=\hat{f}_{i}(N)$.

In order ensure parsimony, the Maximum Likelihood estimation of the NVR for each SDP can be exploited to estimate the number of $\alpha$ terms needed to obtain a good parameterization of $f_{i}$. It is straightforward to show (e.g., Ref. [36]) that the $50 \%$ cutoff frequency $\omega_{i}^{*}$ of each IRW process is linked to the NVR by the relationship $\mathrm{NVR}_{i}=4\left(1-\cos \left(2 \pi \omega_{i}^{*}\right)\right)^{2}$. The corresponding period $T_{i}^{*}=1 / 2 \pi \omega_{i}^{*}$ can be compared to $N$ and the ratio $M_{i}^{*}=N / T_{i}^{*}$ gives an order of magnitude for the degrees of freedom associated with the SDR, as well as of the number of $\alpha$ terms to be used. If $M_{i}^{*} \ll 1$ there will be no need for parameterizing $f_{i}$.

So, after choosing the basis for the parameterization (polynomials, Fourier expansions, radial basis functions), one has simply to compute a number $\left(\propto M_{i}^{*}\right)$ of $\alpha^{i}$ terms for each $f_{i}$, $f_{i, j}$, etc. In the case of polynomial expansions, the final results obtained will be fully in line with the approach of Refs. [7,8] 
while, at the same time, helping to avoid over-fitting. In particular, using $M_{i}^{*}$, one can improve the standard polynomial estimation by using a stepwise procedure to select the optimal subset $u_{j}^{i}, j=1, \ldots, M_{i}^{*}$, that provides the best approximation to the non-parametric $f_{i}$ amongst the larger set of basis elements $u_{j}^{i}, j=1, \ldots, M$, with $M / M_{i}^{*}=2 \rightarrow 4$. An example of this kind can be found in [37], where a linear wavelet functional approximation is adopted and the model structure selection criterion is based on the Predicted Residual Sums of Squares (PRESS) statistic.

\section{Analytical examples}

In this section we consider three examples that show some of the main characteristics of the proposed method. We start first with the Sobol' $g$-function, which illustrates various features of the proposed estimation approach. Sensitivity and metamodelling exercises of different complexity can be designed by tuning both the dimensionality $k$ and the relative importance of the different factors in this function. This then demonstrates the advantages of the pre-sorting strategy, as well as the capabilities of the SDR modelling in dealing with high-dimensional models $(k=100)$.

The second example deals with the function of Oakley and O'Hagan [2]; while the third example involves the metamodelling of the complex Level E model. The subsequent subsections provide a summary of the results obtained in all these examples, while the detailed results are available from the first author.

The computational cost of correctly addressing the different levels of analysis are considered from the standpoints of:

- the FF setting, using the elementary effect test;

- the FP setting, where we only require that the SDR estimation clearly identifies the most important factors with respect to an indistinguishable ensemble of intermediately important and irrelevant main effects;

- the full estimation of all main effects, where we require that no irrelevant main effect is over-estimated (i.e. their estimated $S_{i}$ must be $\ll 1 \%$ ) and

$\max _{i}\left(\mathrm{MSE}_{i}\right)<1 \rightarrow 2$,

where $\mathrm{MSE}_{i}=V\left(f_{i}(N)-f_{i}\right) / V(f)$ is the mean squared error of the SDR estimate of $f_{i}$, normalized by the total variance;

- the full estimation of all second order effects, keeping the same requirements as for the main effects.

\subsection{An analytical example: the Sobol' g-function}

The Sobol' $g$-function is defined as follows:

$Y=\prod_{i=1}^{k} g_{i}\left(X_{i}\right), \quad$ where $g_{i}\left(X_{i}\right)=\frac{\left|4 X_{i}-2\right|+a_{i}}{1+a_{i}}$

with $a_{i} \geqslant 0$ and $X_{i} \sim U(0,1)$. This is a strongly non-linear and non-additive model: it is has been used in the past to test global
SA methods [38]. The value for $k$ can be chosen to analyze the dependence of the method on the number of input factors. Moreover, by tuning the spectrum of parameters $a_{i}$, the relative importance of the $X_{i}$ 's can be modified. The importance of an input factor is higher when $a_{i}$ is small, so factors with $a_{i}=0$ will have the maximum sensitivity indices; while $a_{i}=99$ corresponds to almost null sensitivity.

Two cases with $k=15$ are considered. First, it is necessary to test the performance of the Sobol' samples when the factors are not ordered according to their importance and the subsequent improvement obtained by the pre-sorting strategy based on the elementary effect test. Moreover, in order to evaluate the capability of dealing with high dimensional systems, extended examples at $k=100$ are discussed, adding 85 factors with $a_{i}=99$, i.e. almost dummy factors.

\subsubsection{Case 1}

Consider first the $a_{i}$ spectrum shown in Table $1(k=15)$. This spectrum of $a_{i}$ values provides a model following a Pareto law, with a few important factors and the remaining ones with negligible sensitivity. Moreover, this model has a quite high additive component, since the sum of first order effects covers $75 \%$ of the total output variance. This implies a relatively small contribution of interactions; a quite clear separation of the singular effects; and, in most cases, a faster convergence of the metamodels. Table 1 shows the FF setting: here, we can correctly tackle the FF setting with 128 runs $(8 \cdot(k+1))$ exactly identifying the least important factors. This allows us to presort parameters and arrange the columns of the Sobol' sample in a decreasing order of input factor importance. Table 2 shows that, for this model, the FP setting is very easily handled with and without pre-sorting; while, by introducing the pre-sorting exercise, the computational cost necessary to estimate all the first order $f_{i}$ terms, to the same accuracy as in the non-sorted case, reduces from 1024 to 256; and from 4096 to 1024 for the second order terms. The same improvement can be appreciated also by looking at the convergence rate $N^{-\alpha}$ of the MC integral of $f$, where the 'slope' $\alpha$ rises from 0.91 to 0.95 . Table 2 shows that the metamodel based on the HDMR, truncated at the second order terms, covers more than $97 \%$ of the entire model variance, which implies that this second order truncation can be considered as an excellent metamodel.

Table 3 shows the results of the extended model with 100 factors: we can see that the convergence rates are reduced. Taking $8 \cdot(k+1)=808$ model evaluations to compute the elemen-

Table 1

Case 1: $a_{i}$ spectrum and treatment the FF setting

$\begin{array}{lllllllllllllll}X_{1} & X_{2} & X_{3} & X_{4} & X_{5} & X_{6} & X_{7} & X_{8} & X_{9} & X_{10} & X_{11} & X_{12} & X_{13} & X_{14} & X_{15}\end{array}$ $\begin{array}{llllllllllllllll}a_{i} & 99 & 99 & 4.5 & 4.5 & 99 & 99 & 1 & 99 & 0 & 99 & 1 & 9 & 0 & 9 & 99\end{array}$

FF setting, 128 runs

$S_{T_{i}} 0.000 .000 .020 .020 .000 .000 .140 .000 .470 .000 .140 .010 .470 .010 .00$

$\begin{array}{lllllllllllllll}11 & 12 & 5 & 6 & 13 & 14 & 3 & 15 & 2 & 9 & 4 & 7 & 1 & 8 & 10\end{array}$

ee 0.020 .010 .330 .270 .020 .020 .560 .011 .200 .010 .710 .121 .270 .100 .02

$\begin{array}{lllllllllllllll}11 & 14 & 6 & 5 & 12 & 15 & 2 & 9 & 3 & 13 & 4 & 7 & 1 & 8 & 10\end{array}$

For FF setting we show analytic $S_{T_{i}}$ 's with ranks in bold and (ee) elementary effects with ranks in bold. 
Table 2

Convergence tests for Case 1. Exponent $\alpha$ of the convergence rate $N^{-\alpha}$ of the integral of the $g$-function. Runs required for each analysis; $R^{2}$ of the 1 st and 2nd order metamodels (theoretical value in brackets)

\begin{tabular}{lllll}
\hline Pre-sorting & $\alpha$ & \multicolumn{2}{l}{ Type of analysis } \\
\cline { 3 - 5 } & conv. rate & FP & 1st order HDMR & 2nd order HDMR \\
\hline no & 0.9142 & 64 & 1024 & 4096 \\
& & & $76.8 \%(75.2 \%)$ & $96.4 \%(97.25 \%)$ \\
yes & 0.9521 & \multirow{2}{*}{128} & 256 & 1024 \\
& & & $77.7 \%(75.2 \%)$ & $97.5 \%(97.25 \%)$ \\
\hline
\end{tabular}

Table 3

Convergence tests for Case 1, 100 factors extension. Exponent $\alpha$ of the convergence rate $N^{-\alpha}$ of the integral of the $g$-function. Runs required for each analysis; $R^{2}$ of the 1 st and 2 nd order metamodels (theoretical value in brackets)

\begin{tabular}{lllll}
\hline Pre-sorting & $\alpha$ & \multicolumn{2}{l}{ Type of analysis } \\
\cline { 3 - 5 } & conv. rate & FP & 1st order HDMR & 2nd order HDMR \\
\hline no & 0.82 & 2048 & 8196 & \\
& & & $77.2(75 \%)$ & - \\
yes & 0.85 & 512 & 8196 & \\
& & & $75.4 \%(75 \%)$ & - \\
cut & 0.965 & 64 & 256 & 1024 \\
& & & $76.3 \%(75 \%)$ & $96.4 \%(97.1 \%)$ \\
\hline
\end{tabular}

tary effect tests provides a correct picture of the FF setting. The number of realizations needed to make a good first order metamodel rises to 8196 for both the pre-sorted and the unsorted samples. Using pre-sorting raises the rate of convergence from 0.82 to 0.85 and allows for a reduction in the computational cost for FP from 2048 to 512 realizations. However, pre-sorting is not effective for more rapid $f_{i}$ estimation. On the other hand, cutting the 92 factors identified as irrelevant in FF, allows the full metamodel to converge in 1,024 runs, thus reducing the total cost of the analysis by a factor 4 . Reducing the dimensionality of the problem also allows for an improvement of the convergence 'slope' with respect to the sorted case in Table 2.

\subsubsection{Case 2}

Here, we consider the more difficult spectrum

$a_{1 \leqslant i \leqslant k}=[1,99,0.3,1.5,3,9,1.8,8,0,0,4.5,0.5,0.2,0.01,0]$

that provides a model with 4 input factors sharing the same level of sensitivity. This then decreases gradually for the remaining ones, with the result that only one, out of 15 factors, is really unimportant. Non-additivity and interactions also play a major role in this case, since the first order effects only cover $38 \%$ of the total output variance. Needless to say, this situation presents more difficulties for metamodelling (compare, e.g., the convergence rates in Tables 2 and 4).

The performance of the various steps of the analysis is as follows (see Table 4):

- the FF setting is correctly addressed using 128 model runs;

- the FP setting is correctly addressed using 256 model runs with pre-sorting and no sorting;
Table 4

Convergence tests for Case 2. Exponent $\alpha$ of the convergence rate $N^{-\alpha}$ of the integral of the $g$-function; for the sorted case we show the average over replicated sortings obtained with the elementary effect method and in brackets the value for the 'best' ordering. Runs required for each analysis; $R^{2}$ of the 1 st and 2 nd order metamodels (theoretical value in brackets)

\begin{tabular}{lllll}
\hline Pre-sorting & $\alpha$ & \multicolumn{3}{l}{ Type of analysis } \\
\cline { 3 - 5 } & conv. rate & FP & 1st order HDMR & 2nd order HDMR \\
\hline no & 0.73 & 256 & 2048 & 4096 \\
& & & $39.7 \%(37.9 \%)$ & $69.3 \%(73.2 \%)$ \\
yes & \multirow{2}{*}{$0.72(0.84)$} & 256 & 1024 & 2048 \\
& & & $39.1 \%(37.9 \%)$ & $73.8 \%(73.2 \%)$ \\
\hline
\end{tabular}

Table 5

Convergence tests for Case 2, 100 factors extension. Exponent $\alpha$ of the convergence rate $N^{-\alpha}$ of the integral of the $g$-function. Runs required for each analysis; $R^{2}$ of the 1 st and 2 nd order metamodels (theoretical value in brackets)

\begin{tabular}{lllll}
\hline Pre-sorting & $\alpha$ & \multicolumn{3}{l}{ Type of analysis } \\
\cline { 3 - 5 } & conv. rate & FP & 1st order HDMR & 2nd order HDMR \\
\hline no & 0.613 & 1024 & 16,000 & \\
& & & $45.7 \%(37.9 \%)$ & - \\
yes & 0.702 & 2048 & 8192 & \\
& & & $42.7 \%(37.9 \%)$ & - \\
cut & 0.74 & 256 & 1024 & 2048 \\
& & & $39.1 \%(37.9 \%)$ & $73.8 \%(73.2 \%)$ \\
\hline
\end{tabular}

- the correct estimation of the whole spectrum of $f_{i}$ 's is achieved with 1024 (with pre-sorting) and 2048 (no presorting) runs;

- the correct estimation of the whole spectrum of $f_{i, j}$ 's is achieved with 2048 (with pre-sorting) and 4096 (no presorting) runs;

- the slope of the convergence rates are similar in the presorted (0.72) and non-sorted cases (0.72): this is due to the fact that, in this example, the Pareto law is not satisfied and the elementary effect test cannot detect the optimal ordering (i.e. in ascending order of $a_{i}$ ), which would allow for a much larger slope (0.84).

Extending the model to 100 factors (Table 5), we can see that taking $8 \cdot(k+1)=808$ model evaluations to compute the elementary effect tests, as well as eliminating the 85 factors identified as irrelevant, allows us to reduce the total cost of the analysis by a factor of 8-16 for the first order HDMR. Moreover, in the 100-dimensional case, the convergence slope increases from $0.6-0.7$ to 0.74 , after reducing the dimensionality.

\subsection{The analytic function of Oakley and O'Hagan}

This section considers the results obtained with the analytic function used in [2] and compares the performance of the present SDP method with the Gaussian emulator approach of Ref. [2]. The function has 15 input factors, each with a normal distribution $N(0,1)$, and it has the following analytic form:

$Y=\mathbf{a}_{1}^{\mathrm{T}} \mathbf{X}+\mathbf{a}_{2}^{\mathrm{T}} \sin (\mathbf{X})+\mathbf{a}_{3}^{\mathrm{T}} \cos (\mathbf{X})+\mathbf{X}^{\mathrm{T}} \mathbf{M} \mathbf{X}$. 


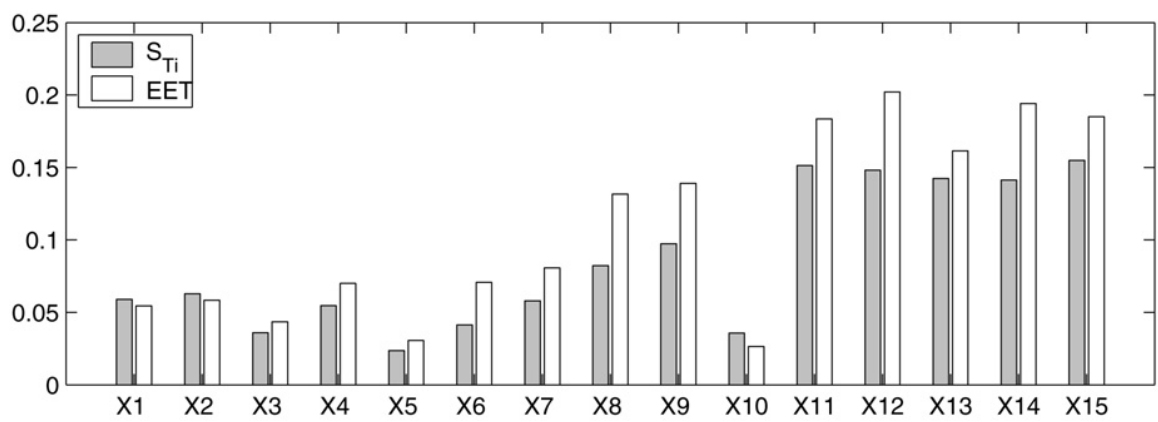

Fig. 5. FF setting for the Oakley-O'Hagan function: analytic total indices $S_{T_{i}}$ and re-scaled elementary effect tests $E E T$ obtained with 128 model evaluations.

Table 6

Convergence tests for the Oakley-O'Hagan function. Exponent $\alpha$ of the convergence rate $N^{-\alpha}$ of the integral of the function. Runs required for each analysis and $\%$ coverage of the metamodel (theoretical coverage in brackets)

\begin{tabular}{lllll}
\hline Pre-sorting & \multirow{2}{*}{ conv. rate } & \multicolumn{2}{l}{ Type of analysis } \\
\cline { 3 - 5 } & & FP & 1st order HDMR & 2nd order HDMR \\
\hline no & 0.89 & 512 & 512 & 2048 \\
& \multirow{2}{*}{$\approx$} & & $68.8 \%(71.1 \%)$ & $96.3 \%(100 \%)$ \\
yes & \multirow{2}{*}{256} & 512 & 1024 \\
& & & $76.2 \%(71.1 \%)$ & $104 \%(100 \%)$ \\
\hline
\end{tabular}

The analysis is based on the same parameter values for the vectors $\mathbf{a}_{i}$ and the matrix $\mathbf{M}$ as those used in [2]. Concerning the FP setting, the 15 input factors can be divided into three groups: $\left[X_{1}-X_{5}\right]$ have a negligible main effect; $\left[X_{6}-X_{10}\right]$ have an 'intermediate' main effect (3-5\%); and $\left[X_{11}-X_{15}\right]$ have the largest main effects (around 10\%). Moreover, the quadratic component of the function produces non-negligible second order interaction terms for any of the 15 input factors. This implies that the none of them can be 'fixed' according to the FF setting (see Fig. 5).

Table 6 presents the convergence tests for the methodology presented in this paper:

- the FF setting is correctly addressed using 128 model realizations;

- the FP setting is correctly addressed using 256 model realizations (pre-sorting) and 512 model realizations (no sorting);

- the correct estimation of the whole spectrum of $f_{i}$ 's is achieved with 512 model realizations (with and without pre-sorting);

- the correct estimation of the $f_{i, j}$ 's (and hence of the full model) needs 1024 model realizations for the pre-sorted case and 2048 for the non-sorted one;

- the slope of the convergence rate is 0.89 for the non-sorted case and rises up to almost 1 with pre-sorting.

In order to compare the results with [2], we can compute the maximum absolute errors for the main effect sensitivity indices estimated by the Gaussian emulator using a maximin LHs sample of 250 model runs, which is as small as 0.007 (second order effects are not analyzed in Ref. [2]). While the SDP approach is able to correctly handle the FP setting with the same number of runs, in order to reach the same precision in the estimation of the sensitivity indices, 1024 realizations are needed using pre-sorting (2048 without pre-sorting). In order to make a clearer comparison, however, we replicated the Gaussian emulator analysis with the same quasi-random sample used for the SDP estimates, using the Gaussian Emulation Machine (GEM) available at http://www.tonyohagan. co.uk/academic/GEM/index.html. Note that GEM analysis is limited to a maximum sample size of 400 , therefore we used the sample size of 256 model evaluations for this comparison. In this case, it can be seen that the precision of GEM and SDP estimates for main effects is very similar, with a maximum absolute error of 0.017 for GEM and 0.019 for SDP. At that same sample size 256, moreover, the maximum absolute error of the GEM estimates of the total effects is 0.0415 , almost identical to the maximum error of the SDP estimates (obtained by summing the first order effect and all second order effects for each factor) which is 0.0419 . Finally, looking in detail at second order effects, the largest analytic value is $2.3 \%$, corresponding to the $\left(X_{1}, X_{11}\right)$ interaction. All the remaining interaction effects are confined between $0 \%$ and $1.7 \%$. For the $\left(X_{1}, X_{11}\right)$ interaction term, the SDP estimate gives $2.6 \%$, which is also clearly distinguishable to be above the estimates of the remaining interaction terms, while GEM gives an estimate of only $1 \%$. Overall, however, neither GEM nor SDP are able to correctly estimate the full pattern of second order effects with this sample size. This is not surprising, since such pattern is not Pareto-like, but presents a bunch of terms in a 'grey' area of small but nonnegligible importance. The absence of a small group of effects clearly dominating above the rest, with the partial exception of the couple $\left(X_{1}, X_{11}\right)$ whose importance is however very small $(2.3 \%)$, impedes to get the full exact metamodel with only 256 model evaluations.

It is worth emphasizing, moreover, that the inclusion of the FF setting in the current approach has a significant added value. Already, at 128 runs, the analysis of the elementary effect tests tells the analyst that none of the 15 parameters has such a small effect that it can be fixed. So, combining this result with the estimation of first order effects only, one already knows that the $\left[X_{1}-X_{5}\right]$ group will have non-negligible interaction effects (and in fact the total effect of the group $\left[X_{1}-X_{5}\right]$ is about $20 \%$ of the total variance of $Y$ ). 
Table 7

List of uncertain input factors for the Level E model

\begin{tabular}{|c|c|c|c|c|}
\hline Notation & Definition & Distribution & Range & Units \\
\hline$X_{1}=T$ & Containment time & Uniform & {$[100,1000]$} & $\mathrm{yr}$ \\
\hline$X_{2}=k_{I}$ & Leach rate for Iodine & Log-Uniform & {$\left[10^{-3}, 10^{-2}\right]$} & $\mathrm{mol} / \mathrm{yr}$ \\
\hline$X_{3}=k_{C}$ & Leach rate for $N p$ chain nuclides & Log-Uniform & {$\left[10^{-6}, 10^{-5}\right]$} & $\mathrm{mol} / \mathrm{yr}$ \\
\hline$X_{4}=v^{(1)}$ & Water velocity in the first geosphere layer & Log-Uniform & {$\left[10^{-3}, 10^{-1}\right]$} & $\mathrm{m} / \mathrm{yr}$ \\
\hline$X_{5}=l^{(1)}$ & Length of the first geosphere layer & Uniform & {$[100,500]$} & $\mathrm{m}$ \\
\hline$X_{6}=R_{I}^{(1)}$ & Retention factor for Iodine in the first layer & Uniform & {$[1,5]$} & - \\
\hline$X_{7}=R_{C}^{(1)}$ & Retention factor for the chain elements in the first layer & Uniform & {$[3,30]$} & - \\
\hline$X_{8}=v^{(2)}$ & Water velocity in the second geosphere layer & Log-Uniform & {$\left[10^{-2}, 10^{-1}\right]$} & $\mathrm{m} / \mathrm{yr}$ \\
\hline$X_{9}=l^{(2)}$ & Length of the second geosphere layer & Uniform & {$[50,200]$} & $\mathrm{m}$ \\
\hline$X_{10}=R_{I}^{(2)}$ & Retention factor for Iodine in the second layer & Uniform & {$[1,5]$} & - \\
\hline$X_{11}=R_{C}^{(2)}$ & Retention factor for the chain elements in the second layer & Uniform & {$[3,30]$} & - \\
\hline$X_{12}=W$ & Stream flow rate & Log-Uniform & {$\left[10^{5}, 10^{7}\right]$} & $\mathrm{m}^{3} / \mathrm{yr}$ \\
\hline
\end{tabular}

Table 8

Maximum absolute errors of normalized SDP sensitivity estimates $S_{i}^{*(N)}=$ $\hat{V}_{i}^{(N)} / \hat{V}^{\text {asympt }}$ for the Level E model over all time points and all input factors. For the pre-sorted case, we show the average over three replicas

\begin{tabular}{lll}
\hline N. or runs & MAE sort & MAE no sort \\
\hline 256 & 0.079 & 0.084 \\
512 & 0.078 & 0.048 \\
1024 & 0.073 & 0.025 \\
2048 & 0.038 & 0.022 \\
4096 & 0.024 & 0.015 \\
8192 & 0.012 & 0.0077 \\
\hline
\end{tabular}

\section{The Level E model}

The Level E model predicts the radiological dose to humans, over geological time scales, due to the underground migration of radionuclides from a nuclear waste disposal site. It has been used both as a benchmark for Monte Carlo computation [39] and as a benchmark for SA methods [40]. See [41] for a review and [1] for an in-depth analysis of the model.

The scenario considered in the model tracks the onedimensional migration of four radionuclides $\left({ }^{129} \mathrm{I}\right.$ and the chain ${ }^{237} \mathrm{~Np} \rightarrow{ }^{233} \mathrm{U} \rightarrow{ }^{229} \mathrm{Th}$ ) through two geosphere layers characterized by different hydro-geological properties. The processes being considered in the model are radioactive decay, dispersion, advection and chemical reaction between the migrating radionuclides and the porous medium. Sometimes, after the steel canister containing the waste has lost its integrity (the time of containment failure is indicated by the factor $T$ ), the release of radionuclides to the geosphere depends only on the leach rates $\left(k_{(\cdot)}\right)$ and the initial inventory levels.

The output quantity of interest $Y(t)$ is the annual radiological dose due to the four radionuclides. The simulated time frame for applications presented here ranges from $6 \cdot 10^{3}$ to $1 \cdot 10^{7}$ years. The predictive uncertainty about $Y(t)$ is due to uncertainties in the twelve input factors, as listed in Table 7.

Amongst the twelve parameters, $X_{4}\left(=v^{(1)}\right)$ and $X_{12}(=W)$ have the largest main effect over the simulated period. The asymptotic values estimated using standard SA tools (Sobol' method) were compared, taking $1,872,000$ realizations, with the
SDP estimation having total costs up to 8192 . After only 1024 runs, which is a very small sample size for this kind of model, the sensitivity pattern is estimated correctly.

The maximum absolute errors of all the main effect sensitivity indices, across all the time co-ordinates considered for the output $Y(t)$, are shown in Table 8 . It is clear that the convergence of the estimates to the asymptotic values is more difficult in this case than in the previous ones. The estimation of the sensitivity patterns are already acceptable between 1024 and 2048 runs and become very good with 4096 runs and higher. Since the dimensionality of the problem is relatively small $(k=12)$ no particular advantages were obtained by pre-sorting.

Comparing SDR total costs with the classical Sobol' sensitivity indices technique, 40,000 model realizations are required to reach an accuracy comparable to the cheaper SDP model estimation of 1024 model runs, i.e. the SDP modelling approach reduces the computational time by a factor 40 in this case. Conversely, 1024 realizations for the Sobol' estimates are much too few, with absolute errors for the sensitivity indices that can reach $0.7-0.8$, i.e. totally unreliable estimates.

\section{Conclusions}

The use of SDP models is a powerful tool for a fast and accurate estimation of truncated HDMR expansions of computational models. All the estimates are performed with a unique sample, which can be any standard Monte Carlo sample. For optimal convergence, the SDP estimation is performed using Sobol' $L P_{\tau}$ quasi-random number generators and is accompanied by a pre-sorting procedure based on the elementary effect test. This allows for the optimal exploitation of the sequential characteristics of $L P_{\tau}$ sequences and significantly faster convergence. Moreover, the pre-sorting procedure can also lead to a reduction of dimensionality, by eliminating irrelevant input factors from the analysis.

We have tested the method with different models and different levels of detail: the FF setting can be tackled efficiently by applying the elementary effect tests at the cost of $8 \cdot(k+1)$ model realizations. For models having up to $10-15$ input factors, 256 runs are sufficient to handle the FP setting, while 
256-1024 runs are needed for correct estimates of the entire spectrum of main effects $f_{i}$. In the case of second order HDMR terms, 1024-2048 realizations are required. The highest computational costs are linked to strong non-additivity and nonmonotonicity of the model, as in the Case $2 g$-function or the Level E model.

The dependence of the computational cost of the method on the number $k$ of input factors is mild for $k$ up to $\sim 20$. In principle, the method can be applied also for very high dimensional systems. We have treated successfully two examples with the number of factors $k=100$. In this case, however, the rate of convergence becomes much smaller and the pre-sorting procedure becomes essential to reduce the dimensionality of the problem.

A comparison with the method of Ref. [2] was also performed on the analytic function used in that paper. The two methods provided very similar results, in terms of maximum absolute errors with respect to analytic values of main and total effects, while SDP performed slightly better in highlighting the most significant two-way interaction.

Finally, we can discuss how far both the FP and FF settings can be tackled by any of the methods in the emulation context. All of them are capable of correctly handling the FP setting (i.e. computing main effects) in an extremely efficient way. This is due to the fact that the correct estimation of the main effects is not affected by the interactions terms of higher order. On the other hand, for any emulator to work properly for the FF setting as well, the hypothesis that the interactions in the model does not have to exceed an order of 2 or 3 is always required. While explicitly stated for the present method and for the cutHDMR and RS-HDMR methods [5,7,8], this hypothesis is also implicit for all other types of emulators. In fact, a few hundreds of Monte Carlo runs are unable to 'activate' high order interactions and so any emulator would, in all cases, reflect only the active interactions up to the $2 \mathrm{nd} / 3 \mathrm{rd}$ order. The consequence of this is that total indices can be underestimated with the risk of wrongly fixing factors. Separately addressing the FF setting is therefore essential and the elementary effect test provides an extremely cheap solution.

\section{Acknowledgements}

The authors are grateful to an anonymous reviewer for some helpful suggestions.

\section{References}

[1] A. Saltelli, S. Tarantola, F. Campolongo, M. Ratto, Sensitivity Analysis in Practice: A Guide to Assessing Scientific Models, John Wiley and Sons, 2004.

[2] J. Oakley, A. O'Hagan, Probabilistic sensitivity analysis of complex models: a Bayesian approach, J. Royal Stat. Soc. B 66 (2004) 751-769.

[3] A. Saltelli, Making best use of model valuations to compute sensitivity indices, Computer Physics Communications 145 (2002) 280-297.

[4] J. Sacks, W.J. Welch, T.J. Mitchell, H.P. Wynn, Design and analysis of computer experiments, Statistical Science 4 (1989) 409-435.

[5] H. Rabitz, O.F. Aliş, J. Shorter, K. Shim, Efficient input-output model representations, Computer Physics Communications 117 (1999) 11-20.
[6] H. Rabitz, O.F. Aliş, Managing the tyranny of parameters in mathematical modelling of physical systems, in: A. Saltelli, K. Chan, M. Scott (Eds.), Sensitivity Analysis, John Wiley and Sons Publishers, 2000, pp. 199-223.

[7] G. Li, S.W. Wang, H. Rabitz, Practical approaches to construct RS-HDMR component functions, Journal of Physical Chemistry 106 (2002) 87218733.

[8] G. Li, J. Hu, S.-W. Wang, P. Georgopoulos, J. Schoendorf, H. Rabitz, Random Sampling-High Dimensional Model Representation (RS-HDMR) and orthogonality of its different order component functions, Journal of Physical Chemistry A 110 (2006) 2474-2485.

[9] P.C. Young, Time variable and state dependent modelling of nonstationary and nonlinear time series, in: T.S. Rao (Ed.), Developments in Time Series Analysis, Chapman and Hall, London, 1993, pp. 374-413.

[10] R. Kalman, A new approach to linear filtering and prediction problems, ASME Trans, Journal Basic Eng. 82D (1960) 35-45.

[11] F. Campolongo, J. Cariboni, A. Saltelli, An effective screening design for sensitivity analysis of large models, Environmental Modelling and Software 22 (2007) 1509-1518.

[12] I. M. Sobol', V. I. Turchaninov, Y. L. Levitan, B. V. Shukhman, Quasirandom sequence generators, Ipm zak. no. 30, Keldysh Institute of Applied Mathematics, Russian Academy of Sciences, Moscow, 1992.

[13] A. Saltelli, M. Ratto, S. Tarantola, F. Campolongo, Sensitivity analysis for chemical models, Chemical Reviews 105 (2005) 2811-2828.

[14] I.M. Sobol', Sensitivity estimates for nonlinear mathematical models, Matematicheskoe Modelirovanie 2 (1990) 112-118 (in Russian); translated in English in [15].

[15] I.M. Sobol', Sensitivity analysis for non-linear mathematical models, Mathematical Modelling and Computational Experiment 1 (1993) 407414 (English translation of Russian original paper [14]).

[16] P.C. Young, Stochastic, dynamic modelling and signal processing: Time variable and state dependent parameter estimation, in: W.J. Fitzgerald, R.L. Smith, A.T. Walden, P.C. Young (Eds.), Nonlinear and Nonstationary Signal Processing, Cambridge University Press, Cambridge, 2000, pp. 74114.

[17] P.C. Young, The identification and estimation of nonlinear stochastic systems, in: A.I. Mees (Ed.), Nonlinear Dynamics and Statistics, Birkhauser, Boston, 2001.

[18] P.C. Young, P. McKenna, J. Bruun, Identification of nonlinear stochastic systems by state dependent parameter estimation, International Journal of Control 74 (2001) 1837-1857.

[19] I.M. Sobol', Uniformly distributed sequences with additional uniformity properties, USSR Comput. Math. Math. Phys. 16 (5) (1976) 236-242.

[20] P. Bratley, H. Fox, H. Niederreiter, Implementation and tests of lowdiscrepancy sequences, ACM Trans. Model. Comput. Simulation 2 (1993) 195-213.

[21] I.M. Sobol', On quasi-Monte Carlo integrations, Mathematics and Computers in Simulation 47 (1998) 103-112.

[22] A. Owen, Latin supercube sampling for very high- dimensional simulations, ACM Transactions on Modeling and Computer Simulation 8 (1998) $71-102$.

[23] I.M. Sobol', B.V. Shukhman, Integration with quasirandom sequences: Numerical experience, International Journal of Modern Physics C 6 (2) (1995) 263-275.

[24] S. Paskov, J. Traub, Faster evaluation of financial derivatives, Journal of Portfolio Management 22 (1995) 113-120.

[25] S. Kucherenko, W. Mauntz, Application of global sensitivity indices for measuring the effectiveness of Quasi-Monte Carlo methods, Monte Carlo Methods and Simulation (2007), in press.

[26] M.D. Morris, Factorial sampling plans for preliminary computational experiments, Technometrics 33 (1991) 161-174.

[27] W. Feller, The fundamental limit theorems in probability, Bull. Amer. Math. Soc. 51 (1945) 800-832.

[28] P.C. Young, C.N. Ng, Variance intervention, Journal of Forecasting 8 (1989) 399-416.

[29] C.N. Ng, P.C. Young, Recursive estimation and forecasting of nonstationary time series, Journal of Forecasting 9 (1990) 173-204. 
[30] P.C. Young, Nonstationary time series analysis and forecasting, Progress in Environmental Science 1 (1999) 3-48.

[31] F. Schweppe, Evaluation of likelihood functions for Gaussian signals, IEEE Trans. on Information Theory 11 (1965) 61-70.

[32] T. Hastie, R. Tibshirani, Generalized Additive Models, Chapman and Hall, 1996.

[33] P.C. Young, D.J. Pedregal, Recursive fixed interval smoothing and the evaluation of Lidar measurements, Environmetrics 7 (1996) 417-427.

[34] P.C. Young, Advances in real-time flood forecasting, Philosophical Trans. Royal Society, Physical and Engineering Sciences 360 (2002) 1433-1450.

[35] K. Doksum, A. Samarov, Nonparametric estimation of global functionals and a measure of the explanatory power of covariates in regression, Annals of Statistics 23 (5) (1995) 1443-1473.

[36] P.C. Young, D. Pedregal, Recursive and en-bloc approaches to signal extraction, Journal of Applied Statistics 26 (1999) 103-128.
[37] N.-V. Truong, L. Wang, P.C. Young, Nonlinear system modelling based on non-parametric identification and linear wavelet estimation of SDP models, International Journal of Control 80 (2007) 774-788.

[38] A. Saltelli, K. Chan, M. Scott (Eds.), Sensitivity Analysis, Wiley Series in Probability and Statistics, John Wiley and Sons, New York, 2000.

[39] OECD/NEA PSAC User group, in: B.W. Goodwin, J.M. Laurens, J.E. Sinclair, D.A. Galson, E. Sartori (Eds.), PSACOIN Level E intercomparison, Nuclear Energy Agency, Organisation for Economic Cooperation and Development, Paris, 1989.

[40] OECD/NEA PSAC User group, in: A. Alonso, P. Robinson, E.J. Bonano, D.A. Galson (Eds.), PSACOIN Level S intercomparison, Nuclear Energy Agency, Organisation for Economic Cooperation and Development, Paris, 1993.

[41] A. Saltelli, S. Tarantola, On the relative importance of input factors in mathematical models: safety assessment for nuclear waste disposal, Journal of American Statistical Association 97 (2002) 702-709. 